\title{
Caracterización energética del carbón vegetal producido en el Norte-Centro de México
}

\section{Energy characterization of charcoal produced in North Central México}

\author{
Carlos De la Cruz Montelongo', Jaime Herrera Gamboa', Ixchel Abby Ortiz Sánchez1', Julio César Ríos Saucedo², \\ Rigoberto Rosales Serna² y Artemio Carrillo-Parra ${ }^{3 *}$
}

1 Tecnológico Nacional de México. Campus Valle del Guadiana. Villa Montemorelos, Durango, México.
2 Instituto Nacional de Investigaciones Forestales,
Agrícolas y Pecuarias. Campo Experimental Valle del
Guadiana. Durango, Durango, México.
3 Universidad Juárez del Estado de Durango. Instituto de Silvicultura e Industria de la Madera. Durango Durango, México

* Autor de correspondencia. acarrilloparra@ujed.mx

\section{RESUMEN}

El carbón vegetal elaborado en México proviene de diferentes especies y procesos, por lo que sus características varían ampliamente. El objetivo de este trabajo fue caracterizar el carbón vegetal producido en el Norte-Centro de México, según normas internacionales de calidad y energía. Se recolectaron al azar tres bolsas de nueve procedencias comerciales de carbón de mezquite (Prosopis sp.), ébano (Ebenopsis sp.) y encino (Quercus sp.). Se determinaron granulometría, contenido de humedad, material volátil, carbono fijo, poder calorífico y los elementos $\mathrm{Mg}, \mathrm{Ca}, \mathrm{Cu}, \mathrm{Zn}$ y Fe. Los datos se sometieron a un análisis de varianza en un diseño completamente aleatorio con cuatro repeticiones por bolsa y comparación de medias con la prueba de Tukey. Predominó el tamaño $20 \mathrm{~mm}>\mathrm{X} \leq 50 \mathrm{~mm}$, únicamente dos procedencias presentaron fragmentos $>100 \mathrm{~mm}$. Los valores promedio fueron: contenido de humedad $5.1 \%$, material volátil $25.9 \%$, ceniza $6.3 \%$ y carbono fijo $62.7 \%$. El poder calorífico más alto fue $27669 \mathrm{~kJ} \mathrm{~kg}^{-1} \mathrm{y}$ se registraron valores elevados de Ca (177 $\left.266.6 \mathrm{mg} \mathrm{kg}^{-1}\right)$ y Mg (20 $\left.263.3 \mathrm{mg} \mathrm{kg}^{-1}\right)$. Las procedencias 5,7 y 8 presentaron más carbono fijo. La variación se relacionó con la especie y procedencia. Es necesario mejorar la calidad del carbón producido en México considerando granulometría, carbono fijo y poder calorífico, así como niveles bajos de contenido de humedad, material volátil y cenizas. La evaluación constante de estos componentes permitirá definir acciones para mejorar la calidad del carbón producido en México y facilitará su comercialización en mercados internacionales.

PALABRAS CLAVE: calidad, carbono fijo, ceniza, granulometría, material volátil, poder calorífico.

\section{ABSTRACT}

The charcoal produced in Mexico comes from different species and processes, so that its characteristics have a wide variation. The objective was to characterize the charcoal produced in the North Central region of México according to international standards of quality and energy. Three bags of nine commercial provenances of mesquite charcoal (Prosopis sp.), ebony (Ebenopsis sp.) and oak (Quercus sp.), were randomly collected. Readings were obtained for particle size, volatile material, moisture content, fixed carbon, calorific value and the elements $\mathrm{Mg}, \mathrm{Ca}, \mathrm{Cu}, \mathrm{Zn}$ and $\mathrm{Fe}$. Data were subjected to analysis of variance under a completely randomized design with four replications per bag and comparison of means were developed with Tukey test. It was found a predominance of $20 \mathrm{~mm}>\mathrm{X} \leq 50 \mathrm{~mm}$ size, only two provenances showed fragments $>100 \mathrm{~mm}$. Mean values were: moisture content $5.1 \%$, volatile material $25.9 \%$, ash $6.3 \%$ and fixed carbon $62.7 \%$. The highest calorific value was $27669 \mathrm{~kJ} \mathrm{~kg}^{-1}$ and higher values for Ca (177 $\left.267 \mathrm{mg} \mathrm{kg}^{-1}\right)$ and Mg $(20263 \mathrm{mg}$ $\left.\mathrm{kg}^{-1}\right)$. Provenances 5, 7 and 8 showed more fixed carbon and volatile material. The variation was related to plant species, provenance as well as the method of production. It is necessary to improve charcoal quality according to particle size, fixed carbon and calorific value; as well as low levels for moisture content, volatile material, and ash content. The constant evaluation of these characteristics will improve the quality of the coal produced in México and facilitate its trading in the international markets.

KEYWORDS: quality, fixed carbon, ash, granulometry, volatile material, calorific value. 


\section{INTRODUCCIÓN}

La energía térmica de la madera se ha empleado desde la antigüedad para satisfacer las necesidades domésticas de cocción de alimentos y calefacción (Ríos, Santos y Gutierrez, 2017). El uso de combustibles a partir de la biomasa favorece el balance de las emisiones de carbono registradas durante la generación de energía. Además, la leña, residuos de la industria maderera y otros tipos de biomasa tienen gran potencial como fuente de energía (Melissari, 2012).

La carbonización de la madera duplica su contenido energético y reduce $75 \%$ el peso original, lo cual facilita su transporte y almacenamiento (Masera et al. 2011). Sin embargo, múltiples factores determinan la calidad del carbón vegetal como las especies vegetales empleadas, método de elaboración, tipo de horno utilizado y pericia del productor (Navas, 1993).

En 2016 la producción de carbón y leña en México fue de $630900 \mathrm{~m}^{3} \mathrm{r}$ (9.4\% de los productos forestales); siendo los principales estados productores de carbón Durango y Sonora (Secretaría del Medio Ambiente y Recursos Naturales [Semarnat], 2017). La producción de carbón está basada en diversas especies forestales como encino, mezquite, huizache, ébano, jabín y machiche, las cuales tienen características que favorecen niveles altos de calidad y rendimiento. Se emplean principalmente hornos tradicionales de tierra tipo parva, fosa, media naranja argentino y colmena brasileño (Antal y Grønli, 2003). También otros factores influyen en el rendimiento y calidad del carbón como el tipo de leña, contenido de humedad, acomodo de la madera en el horno y tiempo elaboración.

En el mercado mexicano se identifica la calidad del carbón vegetal empíricamente al considerar la comercialización a granel o empaquetado, con marca o sin marca, sonido metálico, color en tonos azules brillantes, tamaño y presencia de impurezas (Arias, 2006; García, 2008; Bustamante-García, 2011). Sin embargo, no se establecen criterios claros de clasificación en las dimensiones, contenido de humedad, material volátil, cenizas, carbono fijo, poder calorífico y elementos químicos (Emrich, 2013; Heya, Pournavab, Carrillo-Parra y ColinUrieta, 2014).

\section{OBJETIVOS}

El objetivo del presente estudio fue determinar la calidad comercial y energética de nueve procedencias de carbón vegetal distribuidas en la zona Norte-Centro de México empleando granulometría, análisis químicos proximales y poder calorífico como parámetros de comparación.

\section{MATERIALES Y MÉTODOS}

\section{Obtención de muestras}

Se realizó un muestreo discrecional en establecimientos comerciales en el norte-centro de México, se obtuvieron al azar tres bolsas de carbón vegetal de cada procedencia, seis con registro comercial y tres sin registro. Las muestras obtenidas se codificaron con un número secuencial conforme al momento de su obtención, de esa forma se tuvieron procedencias de la uno a la nueve (Tabla 1). El carbón presentó distinto origen vegetal como mezquite (Prosopis sp.), ébano (Ebenopsis sp.) y encino (Quercus sp.), así como diferente procedencia geográfica y peso.

\section{Granulometría}

La caracterización granulométrica del carbón se estableció mediante tres cribas con aperturas de $20 \mathrm{~mm} \times 20 \mathrm{~mm}, 50$ $\mathrm{mm} \times 50 \mathrm{~mm}$ y $100 \mathrm{~mm} \times 100 \mathrm{~mm}$. Para la evaluación, se apilaron las cribas de mayor a menor apertura con un contenedor en el fondo, se vació la totalidad de la muestra contenida en la bolsa en la criba superior y se agitó continuamente de manera manual durante 30 segundos. La masa retenida en cada criba se separó y pesó individualmente, lo mismo que el residuo que quedó en el fondo del contenedor. Los cálculos se realizaron con base en la relación entre el peso inicial y la masa retenida en cada una de las cribas y el residuo. 
TABLA 1. Información del carbón comercial obtenido en el norte-centro de México.

\begin{tabular}{cccc}
\hline Procedencia & Especie & Procedencia & Peso $(\mathrm{kg})$ \\
\hline 1 & Mezquite & Nuevo León & 3.0 \\
2 & Ébano & Ciudad de México & 2.5 \\
3 & N/D & Nuevo León & 3.0 \\
4 & Ébano y Mezquite & N/D & 2.9 \\
5 & Mezquite & Nuevo León & 3.0 \\
6 & Mezquite & Nuevo León & 5.5 \\
7 & Encino & Durango & 3.0 \\
8 & N/D & Hidalgo & 5.8 \\
9 & N/D & Oaxaca & 2.8 \\
\hline
\end{tabular}

N/D = Información no disponible

TABLA 2. Ecuaciones utilizadas para determinar los análisis proximales de nueve procedencias de carbón vegetal del norte-centro de México.

\begin{tabular}{lcl}
\hline Característica & Ecuación & Descripción de variables \\
\hline Contenido de Humedad (CH) & $\mathrm{CH}=[(\mathrm{A}-\mathrm{B}) / \mathrm{A}] \times 100$ & $\begin{array}{l}\mathrm{A}=\text { Masa inicial de la muestra molida } \\
\text { y cribada }(\mathrm{g})\end{array}$ \\
Material Volátil $(\mathrm{MV})$ & $\mathrm{MV}=[(\mathrm{B}-\mathrm{C}) / \mathrm{B}] \times 100$ & $\begin{array}{l}\mathrm{B}=\text { Masa de la muestra después de } \\
\text { someterla } a 105^{\circ} \mathrm{C}(\mathrm{g})\end{array}$ \\
Contenido de Cenizas $(\mathrm{CC})$ & $\mathrm{CC}=(\mathrm{D} / \mathrm{B}) \times 100$ & $\mathrm{C}=$ Masa de la muestra después de \\
Carbono Fijo (CF) & $\mathrm{CF}=100-\mathrm{CH}-\mathrm{MV}-\mathrm{CC}$ & someterla $a 50^{\circ} \mathrm{C}(\mathrm{g})$
\end{tabular}

\section{Análisis proximales}

Se obtuvieron al azar cuatro muestras representativas del carbón de cada bolsa, las cuales se pulverizaron en un molino marca Retsch ${ }^{\mathrm{TM}} \mathrm{SM}$, con una velocidad de funcionamiento a 1500 revoluciones por minuto (rpm). La molienda se realizó durante un periodo máximo de 3 minutos, para evitar calentamiento y pérdida de material volátil. Se emplearon tamices número $40(425 \mu \mathrm{m})$ y número $60(250 \mu \mathrm{m})$, el material retenido en el tamiz número 60 se usó para realizar los análisis inmediatos según lo establece la norma ASTM D 1762-84 (American Society for Testing and Materials [ASTM], 2013). La determinación del contenido de humedad, material volátil, ceniza y carbono fijo se realizó conforme a la norma e incluyó las ecuaciones mostradas en la tabla 2.

\section{Poder calorífico superior}

El cálculo del poder calorífico superior se realizó con una ecuación que obtiene resultados a partir de la regresión lineal entre el carbono fijo y material volátil (Cordero, Marquez, Rodriguez-Mirasol y Rodriguez, 2001).

$$
\text { PCS }=354.3 \mathrm{CF}+170.8 \mathrm{MV}
$$

Donde:

PCS $=$ poder calorífico superior

$\mathrm{CF}=$ carbono fijo

$\mathrm{MV}=$ material volátil

El carbono fijo representa el carbón (combustible neto) que contiene el mismo carbón vegetal al momento de la combustión, mientras que el material volátil son los gases y 
líquidos piroleñosos que se expulsan desde el carburante al momento de ser sometido a temperaturas altas.

\section{Análisis elemental}

La determinación de cinco elementos (Ca, $\mathrm{Cu}, \mathrm{Fe}, \mathrm{Mg}$ y Zn) en las muestras se realizó con un espectrofotómetro de absorción atómica (Perkin-Elmer, modelo PFBS17030103), siguiendo el procedimiento para material vegetal establecido por el Manual de Métodos Analíticos por espectrofotometría de absorción atómica (Perkin-Elmer Corp., 1996; Chandrasekaran, Hopke, Rector, Allen y Lin, 2012).

\section{Análisis estadístico}

Se utilizó los valores de las cuatro submuestras derivadas de cada una de las nueve procedencias de carbón vegetal. Los resultados porcentuales de los análisis químicos proximales se transformaron con la función de la raíz cuadrada del arcoseno (Scheffler, 1981). Se realizaron pruebas de normalidad Shapiro-Wilks y Kolmogorov para cada variable. Las variables que presentaron normalidad fueron sometidas al análisis de varianza y cuando se encontraron diferencias significativas entre procedencias ( $p \leq 0.05)$, se realizaron comparaciones de medias con la prueba de Tukey. Para las variables que no mostraron normalidad ( $\mathrm{p} \leq 0.05$ ) se aplicó prueba de Kruskal-Wallis. Los datos se procesaron en el programa InfoStat Ver. 2008 (Di Rienzo, 2009).

\section{RESULTADOS Y DISCUSIÓN}

\section{Granulometría}

El resultado de la prueba Shapiro-Wilks demostró la distribución normal de los valores de granulometría ( $\mathrm{p} \geq$ 0.05) (Tabla 3), mientras que el análisis de varianza registró diferencias estadísticas significativas $(\mathrm{p} \leq 0.05)$ entre procedencias y entre tamaño de los trozos de carbón. Las procedencias 8 y 9 fueron las únicas que presentaron fragmentos mayores a $100 \mathrm{~mm}$ (Fig. 1), por otra parte las procedencias 9, 7, 6 y 4 presentaron proporción mayor de fragmentos respectivamente entre $50 \mathrm{~mm}>\mathrm{X} \leq 100 \mathrm{~mm}$ y en la categoría $20 \mathrm{~mm}>\mathrm{X} \leq 50 \mathrm{~mm}$ los porcentajes más altos los presentaron las procedencias 5, 3, 7, 2 y 4. En la categoría $\leq 20 \mathrm{~mm}$ las procedencias que mostraron mayor porcentaje fueron 2, 1, 5 y 8 .

El porcentaje de la clase $20 \mathrm{~mm}>\mathrm{X} \leq 50 \mathrm{~mm}$ y de clases con mayor tamaño fue superior a $80 \%$ en las procedencias 4, 6 , 7 y 9 por lo que se clasifican como "Clase 1" (Bustamante-Garcia, 2011) y pueden ser exportados a España ya que la Norma 13425 de ese país acepta proporciones iguales o superiores a $80 \%$ de fragmentos $>20$ $\mathrm{mm}, 14 \%$ de fragmentos de $10 \mathrm{~mm}$ a $20 \mathrm{~mm}$ y $6 \% \leq 10 \mathrm{~mm}$. Por otra parte, si consideramos los requerimientos de los mercados de Bélgica y Rusia, a las procedencias anteriores se agregaría la procedencia 3, ya que en dichos países se acepta hasta $75 \%$ de fragmentos mayores a $20 \mathrm{~mm}$.

TABLA 3. Prueba de normalidad Shapiro-Wilks en granulometría de carbón vegetal de nueve procedencias.

\begin{tabular}{cccccc}
\hline $\begin{array}{c}\text { Granulometrí } \\
(\mathrm{mm})\end{array}$ & $N$ & Media & D.E & $W^{*}$ & $p$ \\
\hline$\leq 20$ & 27.6 & 10.9 & 0.95 & 0.7747 \\
$20>\mathrm{X} \leq 50$ & & 43.0 & 7.6 & 0.98 & 0.9489 \\
$50>\mathrm{X} \leq 100$ & 33.7 & 13.3 & 0.93 & 0.6093 \\
$>100$ & 1.7 & 3.5 & 0.57 & $<0.0001$ \\
\hline
\end{tabular}

Donde $\mathrm{N}$ = Tamaño de muestra. D.E.= es la desviación estándar. $\mathrm{W}$ = es índice Wilcoxon y p= el valor de significancia. 


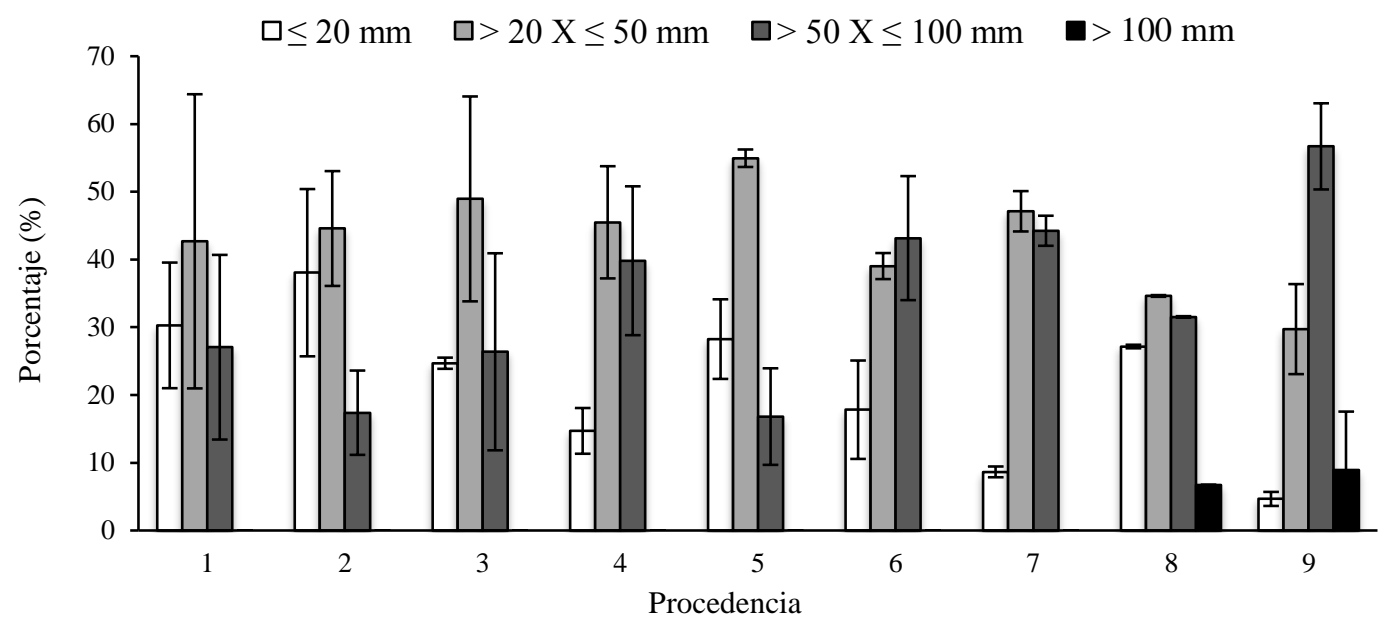

FIGURA 1. Categorías y frecuencia de diferentes tamaños de los fragmentos en nueve procedencias comerciales de carbón vegetal.

TABLA 4. Prueba de normalidad según análisis de Kolmogorov del contenido de humedad, material volátil, cenizas y carbono fijo.

\begin{tabular}{cccccc}
\hline Variable & Media & Varianza & $N$ & $D$ & $p$-valor \\
\hline Humedad & 5.1 & 0.5 & 108 & & $<0.0001$ \\
Material volátil & 25.9 & 25.1 & 108 & 1.00 & $<0.0001$ \\
Cenizas & 6.3 & 3.6 & 108 & 108 & $<0.0001$ \\
Carbono fijo & 62.7 & 22.6 & $<0.0001$ \\
\hline
\end{tabular}

$\mathrm{N}$ = Tamaño de muestra $\mathrm{D}$ = es el estadístico de aproximación asintótica de la distribución y $\mathrm{p}$ = el valor de significancia.

TABLA 5. Análisis Kruskal Wallis del contenido de humedad (\%).

\begin{tabular}{|c|c|c|c|c|c|c|}
\hline Procedencia & $N$ & Medias & D.E. & Medianas & $H$ & $p$ \\
\hline 1 & & 5.0 & 0.5 & 4.8 & & \\
\hline 2 & & 5.2 & 0.4 & 5.0 & & \\
\hline 3 & & 5.3 & 0.3 & 5.1 & & \\
\hline 4 & & 5.0 & 0.3 & 4.7 & & \\
\hline 5 & 12 & 5.0 & 0.2 & 5.0 & 75.1 & $<0.0001$ \\
\hline 6 & & 5.1 & 0.2 & 5.1 & & \\
\hline 7 & & 4.0 & 0.3 & 4.0 & & \\
\hline 8 & & 6.5 & 0.3 & 6.5 & & \\
\hline 9 & & 4.5 & 0.2 & 4.5 & & \\
\hline
\end{tabular}

$\mathrm{N}=$ Tamaño de muestra D.E. = es la desviación estándar. $\mathrm{H}$ = es el valor de la prueba Kruskal Wallis y $\mathrm{p}=$ el valor de significancia

\section{Análisis proximales}

Los porcentajes del contenido de humedad presentaron distribución normal (Tabla 4), los valores mostraron diferencias estadísticas altamente significativas $(\mathrm{p} \leq 0.0001)$ entre procedencias (Tabla 5). El valor más bajo (4.0\%) lo presentó la procedencia 7 , seguida de la procedencia 9
(4.5\%) (Fig. 2a). El resto de las procedencias mostraron valores de humedad entre $5.0 \%$ y $6.5 \%$. Los valores de contenido de humedad de las nueve procedencias fueron relativamente bajos ya que se situaron en un nivel inferior a $7.0 \%$, lo que les permite ser aceptados en mercados internacionales, como el de Bélgica (NBN-M11-001, 1984). 

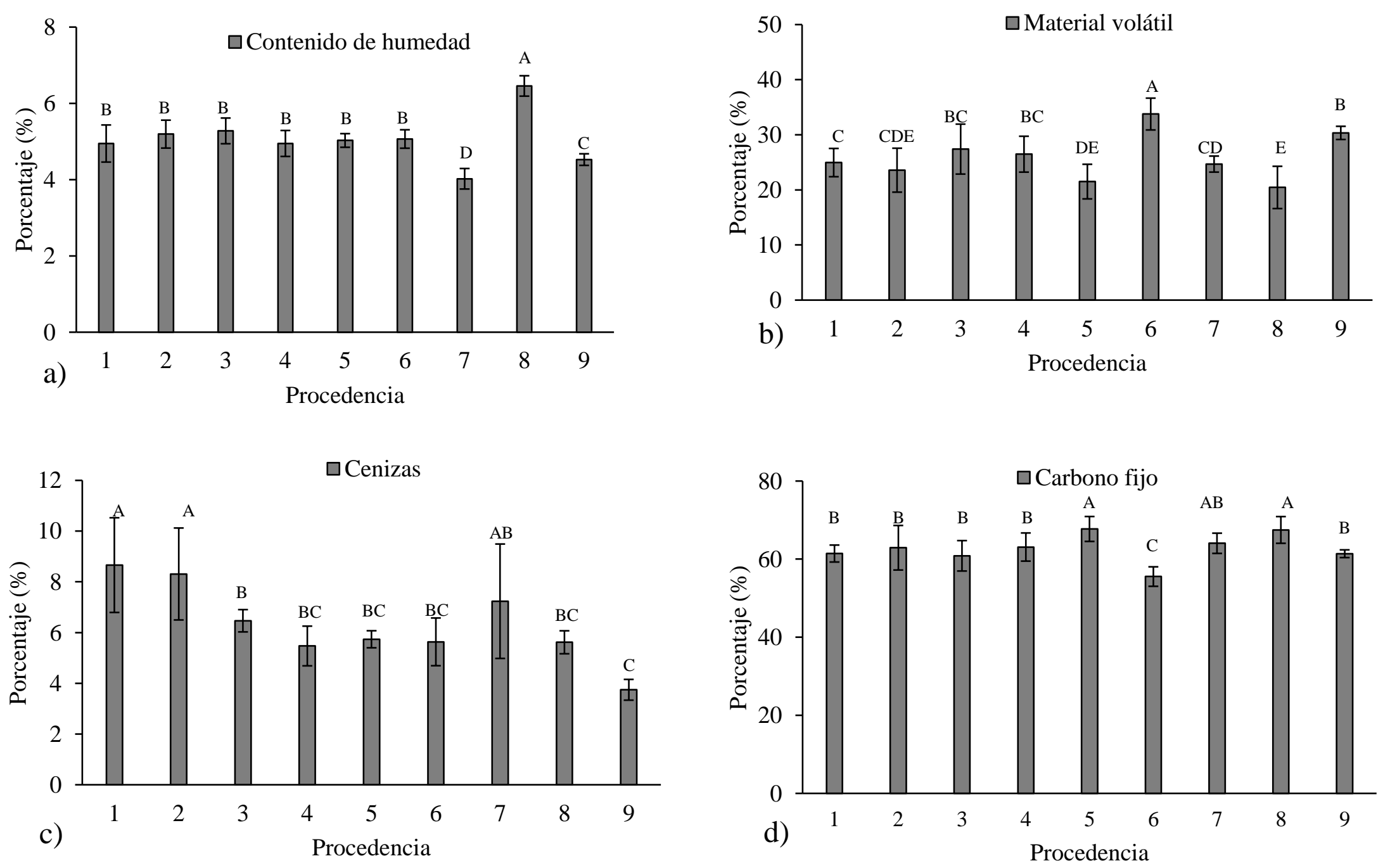

FIGURA 2. Contenido de humedad, material volátil, cenizas y carbono fijo de carbón vegetal de nueve procedencias. Medias con la misma letra son estadísticamente iguales $(\mathrm{p} \leq 0.05)$. 
El contenido de material volátil presentó distribución normal (Tabla 4), los valores mostraron diferencias altamente significativas $(\mathrm{p} \leq 0.0001)$ entre procedencias (Tabla 6). El porcentaje más alto lo presentaron las procedencias $6(33.7 \%)$ y $9(30.3 \%)$; mientras que el valor más bajo (20.4\%) se registró en la procedencia 8 (Fig. 2b). Se han presentado valores similares en el material volátil de carbón vegetal para Prosopis sp. (22.8\%) y Ebenopsis sp. $(24.9 \%)$ (Carrillo-Parra, Foroughbakhch-Pournavab y Bustamante-García, 2013). Los porcentajes de material volátil de alrededor de $15.0 \%$ en carbón vegetal de Quercus sp. se han relacionado con una calidad alta, por la rápida ignición, presencia de flama y producción alta de chispas (García, 2008). Valores bajos de material volátil dificultan el encendido, sin embargo se tendrá una combustión limpia ya que un contenido alto de volátiles produce demasiado humo durante la combustión y se genera más carbonilla (Organización de las Naciones Unidas para la Agricultura y la Alimentacion [FAO], 1983). No obstante de encontrar valores bajos de material volátil en las nueve procedencias, ninguna calificó para el mercado de Bélgica (NBN-M11001, 1984) y Alemania (Deutsches Institut für Normung [DIN], 1989), en los cuales se establece un máximo de $16 \%$ de material volátil.

El contenido de ceniza presentó una distribución normal (Tabla 4), los valores mostraron diferencias altamente significativas $(\mathrm{p} \leq 0.0001)$ entre procedencias (Tabla 7). La procedencia 9 mostró el valor más bajo (3.7\%) (Fig. 2c), niveles similares de contenido de cenizas fueron presentados por Carrillo-Parra et al. (2013) para las especies de Prosopis sp. (2.8\%) y Ebenopsis sp. (3.2\%). Las procedencias 4, 5, 6, 8 y 9 mostraron niveles de ceniza inferiores $6.0 \%$ los cuales se consideran de calidad aceptable (García, 2008) mientras que las procedencias 1, 2 , 3 y 7 presentaron valores $>6.0 \%$, atributo poco deseable en el carbón vegetal, debido a que los residuos se acumulan en los depósitos de calderas, estufas y asadores.

El porcentaje de carbono fijo presentó una distribución normal (Tabla 4), los valores mostraron diferencias altamente significativas $(\mathrm{p} \leq 0.0001)$ entre las procedencias (Tabla 8 ). Las procedencias 5 y 8 como grupo (A) y la 7 como grupo (AB) se consideraron como las mejores al mostrar valores significativamente altos de carbono fijo $(67.5 \%-67.7 \%)$ (Fig. 2d). El carbono fijo se considera como una de las características más importantes de los materiales combustibles, debido a que determina la calidad y cantidad de brazas que se forman durante la combustión. De acuerdo con los resultados obtenidos ninguna de las procedencias cumplió con este requisito de calidad según las normas internacionales NBN M11-001, NF Núm. 846 y DIN 51749.

TABLA 6. Análisis Kruskal Wallis del contenido de material volátil (\%).

\begin{tabular}{ccccccc}
\hline Procedencia & $N$ & Medias & D.E. & Medianas & $H$ & $p$ \\
\hline 1 & & 25.0 & 2.6 & 25.7 & & \\
2 & 23.6 & 4.0 & 25.0 & & \\
3 & 27.4 & 4.5 & 27.6 & & \\
4 & & 3.3 & 25.9 & & \\
5 & 12 & 26.5 & 3.1 & 22.7 & 65.9 & \\
6 & & 2.5 & 33.8 & & \\
7 & & 33.8 & 1.5 & 24.3 & & \\
8 & 24.7 & 3.8 & 19.9 & & \\
9 & 20.5 & 1.2 & 30.8 & & \\
\hline
\end{tabular}

$\mathrm{N}=$ Tamaño de muestra D.E.= es la desviación estándar. $\mathrm{H}$ = es el valor de la prueba Kruskal Wallis y $\mathrm{P}=$ el valor de significancia. 
TABLA 7. Análisis Kruskal Wallis del contenido de ceniza (\%).

\begin{tabular}{ccccccc}
\hline Procedencia & $N$ & Medias & D.E. & Medianas & $H$ & $p$ \\
\hline 1 & 8.7 & 1.9 & 9.5 & & \\
2 & 8.3 & 1.8 & 7.6 & & \\
3 & 6.5 & 0.4 & 6.4 & & \\
4 & 5.5 & 0.8 & 5.6 & 6.0001 \\
5 & 12 & 5.7 & 0.3 & 5.7 & & \\
6 & & 5.6 & 0.9 & 5.6 & \\
7 & 7.2 & 2.3 & 7.5 & \\
8 & 5.6 & 0.5 & 5.6 & \\
9 & 3.7 & 0.4 & 3.7 & & \\
\hline
\end{tabular}

$\mathrm{N}$ = Tamaño de muestra D.E.= es la desviación estándar. $\mathrm{H}=$ Estimadores el valor de la prueba Kruskal Wallis y p= el valor de significancia.

TABLA 8. Análisis Kruskal Wallis carbono fijo.

\begin{tabular}{ccccccc}
\hline Muestra & $N$ & Medias & D.E. & Medianas & $H$ & $p$ \\
\hline 1 & 61.4 & 2.2 & 60.5 & & \\
2 & 62.9 & 5.7 & 3.9 & 61.9 & & \\
3 & 60.9 & 3.6 & 61.1 & & \\
4 & 12 & 63.1 & 3.2 & 64.7 & & \\
5 & 67.7 & 2.5 & 66.7 & 56.8 & $<0.0001$ \\
6 & & 2.6 & 55.3 & & \\
7 & 55.5 & 3.4 & 63.1 & & \\
8 & 64.1 & 1.0 & 68.0 & & \\
9 & 67.5 & 61.4 & 61.4 & & \\
\hline
\end{tabular}

$\mathrm{N}$ = Tamaño de muestra D.E.= es la desviación estándar. $\mathrm{H}=$ Estimadores el valor de la prueba Kruskal Wallis y p= el valor de significancia.

\section{Poder calorífico}

El poder calorífico superior presentó diferencias altamente significativas ( $\mathrm{p} \leq 0.0001)$ entre procedencias (Tabla 9). La procedencia 5 (mezquite) mostró el valor más alto con $27669 \mathrm{~kJ} \mathrm{~kg}^{-1}$, mientras que la procedencia 4 (ébano y mezquite) registró un valor de $26879 \mathrm{~kJ} \mathrm{~kg}^{-1}$ (Fig. 3). Dichos valores se pueden considerar bajos en comparación con $30241 \mathrm{~kJ} \mathrm{~kg}^{-1}$ registrado para carbón de mezquite (Prosopis sp.) y $29725 \mathrm{~kJ} \mathrm{~kg}^{-1}$ para ébano (Ebenopsis sp.) (Carrillo-Parra et al., 2013) (Tabla 9)

\section{Elementos}

Los elementos calcio, cobre, fierro, magnesio y zinc son de importancia durante la combustión por la proporción en la ceniza, punto de fusión e inocuidad de los residuos al momento de su dispersión al ambiente. El análisis de varianza mostró diferencia estadística altamente significativa $(\mathrm{p} \leq 0.0001)$ entre procedencias (Tabla 10).

Los resultados de la concentración de $\mathrm{Mg}$ fluctuaron entre $9196 \mathrm{mg} \mathrm{kg}^{-1}(0.9 \%)$ y $20263.3 \mathrm{mg} \mathrm{kg}^{-1}(2.3 \%)$, lo que coincide con Fengel y Wegener (1984) y Orihuela et al. (2016) en su microanálisis en cenizas de materiales lignocelulósicos con valores entre $4832 \mathrm{mg} \mathrm{kg}^{-1} \mathrm{y}$ $39287.1 \mathrm{mg} \mathrm{kg}{ }^{-1}$. La procedencia 4 presentó valores significativamente altos (20 $\left.263 \mathrm{mg} \mathrm{kg}^{-1}\right)$, seguida por las procedencias 5 (19 $017 \mathrm{mg} \mathrm{kg}^{-1}$ ) y 9 (19 $073 \mathrm{mg}$ $\left.\mathrm{kg}^{-1}\right)$ (Fig. 4). 
TABLA 9. Análisis Kruskal Wallis poder calorífico.

\begin{tabular}{cccccc}
\hline Procedencia & $N$ & Medias & $D . E$ & Mediana & $H$ \\
\hline 1 & 26029 & 494.9 & 26046.0 & & \\
2 & 26319 & 1378.2 & 26288.5 & & \\
3 & 26240 & 613.5 & 26348.2 & & \\
4 & 12 & 749.4 & 27308.7 & & \\
5 & 26879 & 604.6 & 27523.3 & 53.7 & $<0001$ \\
6 & 27669 & 439.6 & 25404.3 & & \\
7 & 25443 & 817.1 & 26428.0 & & \\
8 & 26911 & 564.8 & 27481.3 & & \\
9 & 27400 & 178.3 & 26945.2 & & \\
\hline
\end{tabular}

$\mathrm{N}=$ Tamaño de muestra. D.E. = es la desviación estándar. $\mathrm{H}$ = es el valor de la prueba Kruskal Wallis y $\mathrm{p}$ = el valor de significancia.

TABLA 10. Análisis de varianza de contenido elemental entre procedencias.

\begin{tabular}{|c|c|c|c|}
\hline Elemento & Fuente de variación & Procedencia & Error \\
\hline \multirow{4}{*}{ Magnesio } & SC & $5.04 \mathrm{E}+08$ & $1.19 \mathrm{E}+\mathrm{O} 6$ \\
\hline & $\mathrm{CM}$ & 302919 & 88 \\
\hline & $F$ & 3455 & \\
\hline & $\mathrm{p}$-valor & $<0.0001$ & \\
\hline \multirow{4}{*}{ Calcio } & SC & $1.23 E+10$ & $2.01 \mathrm{E}+08$ \\
\hline & $\mathrm{CM}$ & 1536872500 & $1.11 \mathrm{E}+07$ \\
\hline & $\mathrm{F}$ & 137.93 & \\
\hline & $\mathrm{p}$-valor & $<0.0001$ & \\
\hline \multirow{4}{*}{ Cobre } & SC & $9.23 \mathrm{E}+03$ & 7.80E-01 \\
\hline & CM & 1,153 & 0.04 \\
\hline & $F$ & 26,543 & \\
\hline & $\mathrm{p}$-valor & $<0.0001$ & \\
\hline \multirow{4}{*}{ Zinc } & SC & $1.05 \mathrm{E}+05$ & $5.64 \mathrm{E}+\mathrm{O} 1$ \\
\hline & CM & 13,133 & 3.13 \\
\hline & $F$ & 4,192 & \\
\hline & $\mathrm{p}$-valor & $<0.0001$ & \\
\hline \multirow{4}{*}{ Fierro } & SC & $2.42 \mathrm{E}+06$ & $1.58 \mathrm{E}+03$ \\
\hline & CM & 302,919 & 87.69 \\
\hline & $\mathrm{F}$ & 3,455 & \\
\hline & p-valor & $<0.0001$ & \\
\hline
\end{tabular}

SC= Suma de Cuadrados. CM= Cuadrado Medio. F= Valor de igualdad de varianzas. $\mathrm{p}=$ Nivel de significancia. 


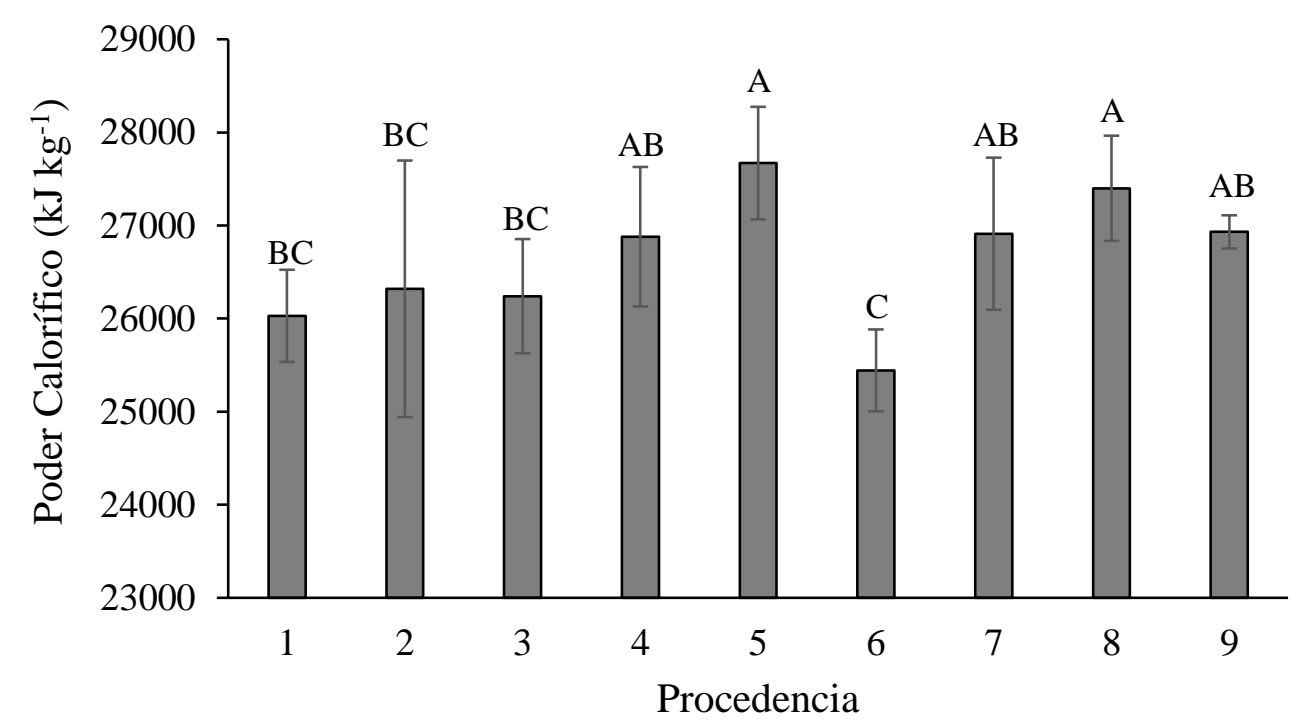

FIGURA 3. Poder calorífico determinado de nueve procedencias comerciales de carbón. Medias con la misma letra son estadísticamente iguales (Tukey $\mathrm{p} \leq 0.05$ ).

La concentración de Ca presentó diferencias estadísticas entre procedencias $(\mathrm{p} \leq 0.05)$. La procedencia 5 mostró un valor significativamente alto con $177266.6 \mathrm{mg} \mathrm{kg}^{-1}$, seguido de las procedencias 1,7 y 9 con valores entre $114333.3 \mathrm{mg}$ $\mathrm{kg}^{-1}$ y $122067.6 \mathrm{mg} \mathrm{kg}^{-1}$ (Fig. 4). Se han registrado variaciones en la concentración de Ca entre $8000 \mathrm{mg} \mathrm{kg}^{-1} \mathrm{y}$ $200000 \mathrm{mg} \mathrm{kg}^{-1}$ en cenizas de maderas duras como se considera al encino (Alakangas, Valtanen y Levlin, 2006; Telmo, Lousada y Moreira, 2010), y hasta $115040 \mathrm{mg} \mathrm{kg}^{-1}$ en el estudio de cenizas de materiales lignocelulósicos realizado por Orihuela, et al. (2016). La concentración alta de $\mathrm{Ca}$, combinada con el punto de fusión bajo $\left(839^{\circ} \mathrm{C}\right)$, genera problemas operativos en el equipo industrial (Correa-Méndez et al., 2014).

Las concentraciones de $\mathrm{Cu}$ en las procedencias de carbón evaluadas fueron bajas, los valores más altos los mostraron las procedencias $3\left(65.4 \mathrm{mg} \mathrm{kg}^{-1}\right)$ y $5(72.0 \mathrm{mg}$ $\left.\mathrm{kg}^{-1}\right)$, mientras que el valor más bajo (19.4 mg kg-1) lo registró la procedencia 7 (Fig. 4). Se han presentado valores muy variados para la concentración de $\mathrm{Cu}$ desde $2.0 \mathrm{mg}$ $\mathrm{kg}^{-1}$ hasta niveles de $520.0 \mathrm{mg} \mathrm{kg}^{-1}$ en ceniza de madera (Alakangas et al., 2006; Telmo et al., 2010).
La procedencia 7 presentó una concentración baja (39.97 $\mathrm{mg} \mathrm{kg}^{-1}$ ) en $\mathrm{Zn}$, mientras que la procedencia 8

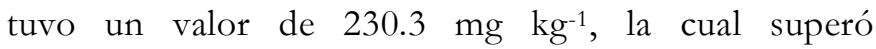
significativamente al resto de las procedencias evaluadas (Fig. 4). Los valores fueron similares a lo registrado en ceniza de materiales lignocelulósicos de $226.2 \mathrm{mg} \mathrm{kg}^{-1}$ hasta $2121.0 \mathrm{mg} \mathrm{kg}^{-1}$ (Orihuela et al., 2016), los valores coinciden en concentraciones reportadas en carbón vegetal puesto que se realizó en cenizas y la carbonización generalmente se realiza a bajas temperatura lo que evita variaciones en la concentración de elementos químicos (Obernberger y Thek, 2004). Así mismo en cenizas de madera de sauce (Salix sp.) (0.3\% - 0.8\%; 3.0 $\mathrm{mg} \mathrm{kg-1}-8.0 \mathrm{mg} \mathrm{kg}^{-1}$ ) y encino (Quercus sp.) (0.07\% $0.5 \%$; $\left.0.7 \mathrm{mg} \mathrm{kg}^{-1}-5.0 \mathrm{mg} \mathrm{kg}^{-1}\right)$ se obtuvieron valores diferentes a los obtenidos en el presente estudio (Fengel y Wegener, 1984; Orihuela et al., 2016). En otro estudio de cenizas de Ocote presentaron valores entre $400 \mathrm{mg}$ $\mathrm{kg}^{-1}$ y $2300 \mathrm{mg} \mathrm{kg}^{-1}$ para $\mathrm{Zn}$ (Chandrasekaran et al., 2012). 


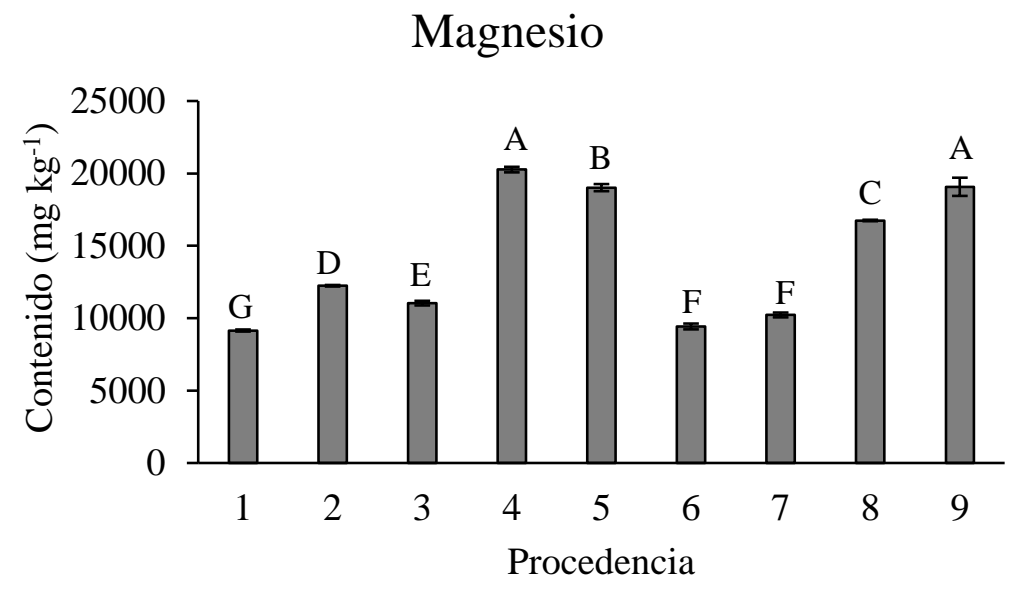

Calcio
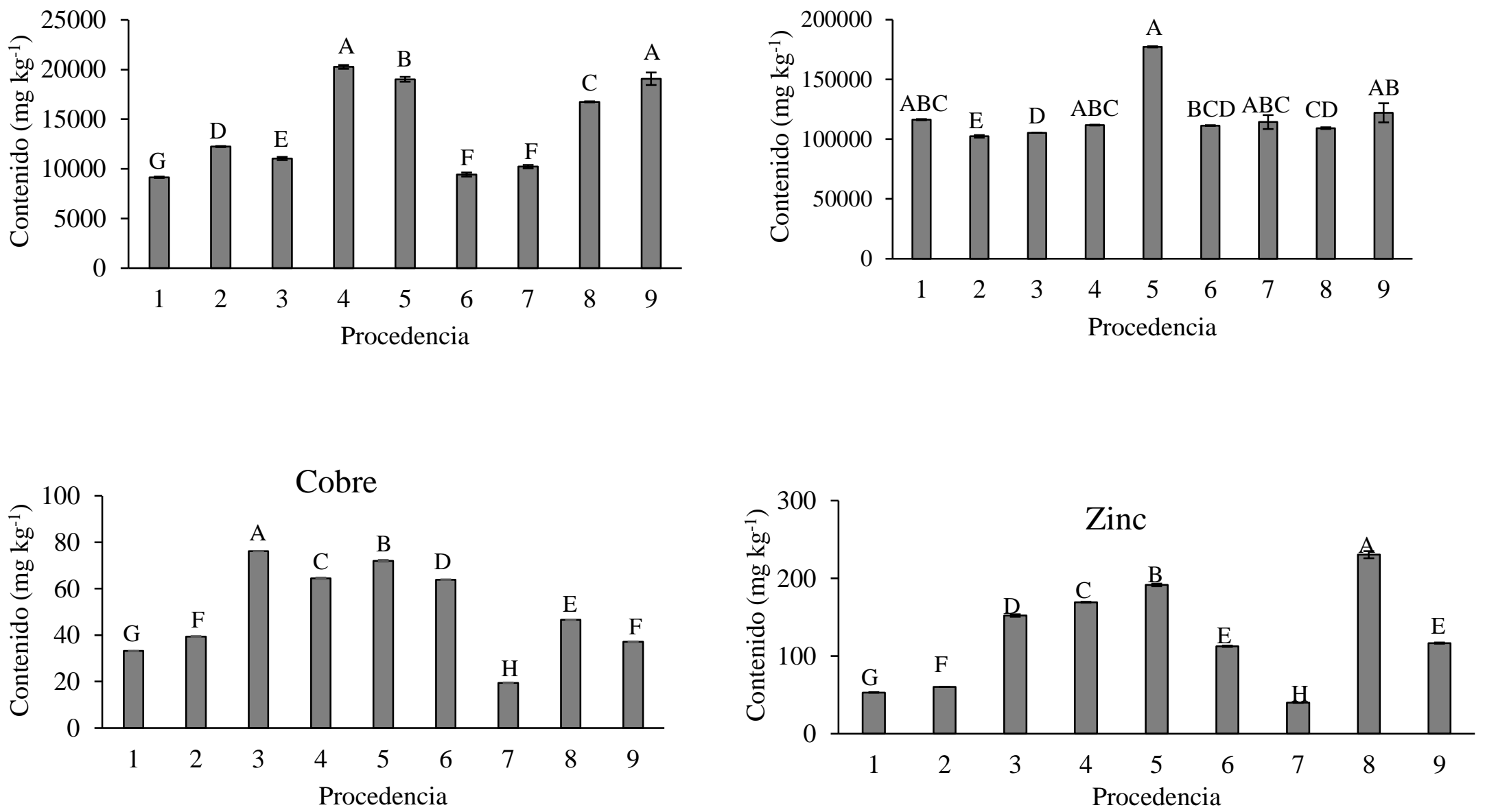

FIGURA 4. Elementos que componen las cenizas de nueve procedencias comerciales de carbón vegetal. Medias con la misma letra son esta dísticamente iguales (Tukey $\mathrm{p} \leq 0.05)$. 
La concentración Fe fue significativamente baja en la procedencia 7 (encino) con $184.7 \mathrm{mg} \mathrm{kg}^{-1}$, mientras que el valor más alto lo presentó la procedencia 1 (mezquite) con $1204 \mathrm{mg} \mathrm{kg}^{-1}$ (Fig. 4). En estudios de cenizas también se observaron valores más altos para Fe, con niveles de hasta $3712 \mathrm{mg} \mathrm{kg}^{-1}$ en cenizas de Pinus sp. (Orihuela et al., 2016).

\section{CONCLUSIONES}

El carbón vegetal debe presentar valores de material volátil $\leq 16 \%$, cenizas $\leq 6.0 \%$, niveles de carbono fijo $>75 \%$ y poder calorífico $>30000 \mathrm{~kJ} \mathrm{~kg}^{-1}$ de manera que permita cumplir los requerimientos de la mayoría de los mercados. Los atributos del carbón producido en la región nortecentro de México son diferentes y algunas procedencias alcanzaron los requerimientos de los mercados internacionales. La distribución granulométrica, contenido de humedad, material volátil, contenido de ceniza, carbono fijo, poder calorífico y porcentaje de elementos en las cenizas fue diferente entre las procedencias del nortecentro de México. La variación se relacionó con las especies vegetales reportadas, destacando con mejores parámetros de calidad el carbón producido con mezquite y/o encino. Respecto a la dimensión del carbón es necesario incrementar la proporción de los tamaños iguales o superiores a $20 \mathrm{~mm} \geq X \leq 50 \mathrm{~mm}$ y así tener una categoría en calidad buena, aun así excede un cierto porcentaje en carbón vegetal de baja calidad. Para usos industriales las concentraciones de elementos en las cenizas de carbón vegetal se deben de mantener bajos para evitar la corrosión y problemas en los equipos. La evaluación constante de las características de calidad y energéticas permitirá implementar acciones para mejorar la calidad del carbón producido en el norte-centro de México y se facilitará su comercialización con mejores productos en los mercados nacionales y de exportación.

\section{RECONOCIMIENTOS}

Se agradece el apoyo del Fondo de Sustentabilidad Energética a través del proyecto SENER CONACYT 2014 246911 Clúster de Biocombustibles Sólidos para la
Generación Térmica y Eléctrica por el apoyo brindado para realizar la colecta y el análisis de las muestras en laboratorio.

\section{REFERENCIAS}

Alakangas, E., Valtanen, J., \& Levlin, J. E. (2006). CEN technical specification for solid biofuels-Fuel specification and classes. Biomass and Bioenergy, 30(11), 908-914. doi: 10.1016 /j.biombioe.2006.06.001

American Society for Testing and Materials [ASTM] (2013). D 1762-84 Standard test method for chemical analysis of wood charcoal. West Conshohocken, PA: ASTM International.

Antal, M.J. \& Grønli, M. (2003). The art, science, and technology of charcoal production. Industrial \& Engineering Chemistry Research, 42(8), 1619-1640. doi: 10.1021/ie0207919

Arias, T. (2006). Estudio de mercado regional de carbón vegetal en Tabasco. CONAFOR-NYDE AC. Inf. Técnico. Querétaro. www.rembio.org.mx

Bustamante-García, V. (2011). Evaluación del proceso de producción de carbón vegetal de residuos de Quercus sideroxyla bumb \& bonpl., en hornos tipo colmena brasileño. Tesis de maestría, Universidad Autónoma de Nuevo León, Linares, México.

Carrillo-Parra, A., Foroughbakhch-Pournavab, R., \& BustamanteGarcía, V. (2013). Calidad del carbón de Prosopis laevigata (Humb. \& Bonpl. ex Willd.) MC Johnst. y Ebenopsis ebano (Berland.) Barneby \& JW Grimes elaborado en horno tipo fosa. Revista Mexicana de Ciencias Forestales, 4(17), 62-71.

Chandrasekaran, S. R., Hopke, P. K., Rector, L., Allen, G., \& Lin, L. (2012). Chemical composition of wood chips and wood pellets. Energy and Fuels, 26(8), 4932-4937. doi: 10.1021/ef300884k

Cordero, T., Marquez, F., Rodriguez-Mirasol, J., \& Rodriguez, J. J. (2001). Predicting heating values of lignocellulosics and carbonaceous materials from proximate analysis. Fuel, 80(11), 1567-1571. doi: 10.1016/S0016-2361(01)00034-5

Correa-Méndez, F., Carrillo-Parra, A., Rutiaga-Quiñones, J. G., Márquez-Montesino, F., González-Rodríguez, H., Jurado-Ybarra, E., \& Garza-Ocañas, F. (2014). Moisture and inorganic substance content in pine timber products for use in pellets and briquettes. Revista Chapingo Series Ciencias Forestales y del Ambiente, 20(1), 77-88. doi: 10.5154/r.rchscfa.2013.04.012

Deutsches Institut für Normung [DIN] (1989). DIN 51749 Testing of solid fuels- Grill charcoal and grill charcoal briquettes: Requirements and test methods. 
Di Rienzo, J. A. (2009). InfoStat versión 2009. Grupo InfoStat, FCA, Universidad Nacional de Córdoba, Argentina. Recuperado de http//www. infostat. com. ar

Emrich, W. (2013). Handbook of charcoal making: the traditional and industrial methods (Vol. 7). Springer Science \& Business Media. doi: 10.1007/978-94-017-0450-2.

Fengel, D. \& Wegener,W G. (1984). Wood chemistry, ultrastructure, reactions. Walter de Gruyter, Berlin and New York. doi: 10.1002/pol.1985.130231112.

García, M. (2008). Carbón de encino: fuente de calor y energía. CONABIO. Biodiversitas, 77, 7-9.

Heya, M. N. Pournavab, F. R., Carrillo-Parra, A., \& Colin-Urieta, S. (2014). Bioenergy potential of shrub from native species of northeastern Mexico. International journal of agricultural policy and research, 2(12), 475-483. doi: 10.15739/IJAPR.020.

Masera, O., Coralli, F., García, C., Riegelhaupt, E., Arias, T., Vega, J., Díaz, R., Guerrero, G., \& Cecotti, L. (2011). La bioenergía en México. Situación actual y perspectivas. México: Red Mexicana de Bioenergía, A.C.

Melissari, B. (2012). Comportamiento de cenizas y su impacto en sistemas de combustión de biomasa. Memoria Investigaciones en Ingeniería, 10, 69-82.

Navas, S. (1993). Calidad del carbón vegetal: evaluación preliminar. Tecnología en Marcha, 12(1), 99-106.

NBN-MII-001 (1984). Charbon de bois et briquettes decharbon de bois á usage domestique: Denomination, Spécifications, Essais.

Obernberger, I. \& Thek, G. (2004). Physical characterisation and chemical composition of densified biomass fuels with regard to their combustion behaviour. Biomass and bioenergy, 27(6), 653-669. doi: 10.1016/j.biombioe.2003.07.006

Organización de las Naciones Unidas para la Agricultura y la Alimentacion [FAO] (1983). Métodos simples para fabricar carbón vegetal. Roma, Italia: FAO.

Orihuela., R., Reyes R., L. A., Rangel M., J. R., Chavez P., M. C., Márquez M., F., Correa M., F., Carrillo-Parra., A., \& Rutiaga-Quiñones., J. (2016). Elaboración de briquetas con residuos maderables de latifoliadas. En J. G. Rutiaga-Quiñones y A. Carrillo-Parra (Eds.), Química de los materiales lignocelulósicos y su potencial bioenergético (pp. 204-217). Sierke Verlag
Perkin-Elmer Corp. (1996). Analytical methods for atomic absorption spectroscopy (Perkin-Elmer Corp.) (pp. 299-237). EUA: Perkin-Elmer Corp.

Ríos B, I. M., Santos C, J., \& Gutiérrez, A. C. (2017). Biocombustibles sólidos: una solución al calentamiento global. Revista Ciencia, 68(4), $1-17$.

Scheffler, W. C. (1981). Bioestadística. México, D.F.: Fondo Educativo Interamericano.

Secretaría del Medio Ambiente y Recursos Naturales [Semarnat]. (2017). Anuario Estadístico de la Producción Forestal. Cd. de México.

Telmo, C., Lousada, J., \& Moreira, N. (2010). Proximate analysis, backwards stepwise regression between gross calorific value, ultimate and chemical analysis of wood. Bioresource technology, 101(11), 3808-3815. doi: 10.1016/j.biortech.2010.01.021.

Manuscrito recibido el 19 de junio de 2019

Aceptado el 2 de octubre de 2019

Publicado el 23 de abril de 2020

Este documento se debe citar como:

De la Cruz M., C., Herrera G., J., Ortiz S., I. A., Ríos S., J. C., Rosales S., R., \& Carrillo-Parra, A. (2020). Caracterización energética del carbón vegetal producido en el NorteCentro de México. Madera y Bosques, 26(2), e2621971. doi: 10.21829/myb.2020.2621971

Madera y Bosques por Instituto de Ecología, A.C. se distribuye bajo una Licencia Creative Commons Atribución-NoComercialCompartirlgual 4.0 Internacional. 